\title{
Duplex Ventral Pancreas
}

\author{
Farooq P. Agha \\ Department of Radiology, University of Michigan Medical Center, Ann Arbor, Michigan, USA
}

\begin{abstract}
Complete duplication of the ventral pancreatic ductal system in 2 patients is reported. Both patients, during evaluation for recurrent abdominal pain, underwent endoscopic retrograde cholangiopancreatography that revealed typical changes of chronic pancreatitis and pseudocysts confined to 1 ductal system with the other ductal system completely normal. Both ductal systems filled with contrast medium via a common opening at the major papilla. A rudimentary minor papilla was present, but cannulations were unsuccessful. This unusual anomaly of the ventral pancreas with its embryologic basis, diagnosis, and clinical implications is discussed.
\end{abstract}

Key words: Pancreas, ductal anomalies - Ventral pancreas, duplication.

Pancreas develops from ventral and dorsal anlagen, both of which arise from the caudal portion of the foregut in a 3-4-week-old embryo. The dorsal anlage is the first to arise from the endodermal epithelium of the duodenum and lies opposite and cranial to the hepatic diverticulum. It grows rapidly and extends into the dorsal mesentery of the duodenum. The ventral anlage, consisting of 2 separate buds, develops slightly later and is located caudal to the hepatic diverticulum. The smaller left ventral bud usually regresses and the right bud persists. The ventral anlage undergoes $90^{\circ}$ clockwise rotation and migrates dorsally, eventually occupying a position within the mesoduodenum along the posteroinferior margin of the dorsal pan-

Address reprint requests to: Farooq P. Agha, M.D., Department of Radiology, Evanston Hospital-Northwestern University, 2650 Ridge Avenue, Evanston, IL 60201, USA creatic anlage. Fusion of dorsal and ventral pancreas anlagen at 7-8 weeks of embryonic life results in a mature pancreas [1]. If the 2 pancreatic anlagen do not fuse, the congenital anomaly of pancreas divisum results, in which the duct of Santorini serves as the main drainage duct, and the duct of Wirsung remains small and drains only part of the head via the major papilla $[1,2]$.

The anomaly in our 2 patients showed 2 main pancreatic ducts both draining through the major papilla. Since a rudimentary minor papilla was present, it is believed that this anomaly represents a duplication of the ventral pancreatic ductal system (duct of Wirsung). It is speculated that the ventral pancreas developed into 2 complete ductal systems both draining via the major papilla and probably provided drainage to the entire pancreas.

\section{Case Reports}

\section{Case 1}

A 67-year-old white woman complained of recurrent abdominal pain and nausea of 3 months duration. Enlargement of the tail of the pancreas was noted at computed tomography (CT). A tumor was suspected. She underwent distal pancreatectomy and splenectomy. The histopathologic examination revealed interstitial fibrosis. No malignancy or degenerative changes were found. Three weeks after initial surgery she developed enlarging pseudocyst in the left upper quadrant which was drained percutaneously. This collection continued to regress in size and she was discharged.

She was readmitted 2 months later with recurrent abdominal pain, nausea, vomiting, and fever. A sonogram revealed a pseudocyst in the left upper quadrant that was larger than on the previous studies. At this time she was referred to University Hospital for evaluation.

Physical examination was unremarkable except for tenderness in the epigastrium. Laboratory data revealed slightly elevated serum amylase levels. An ultrasound scan revealed a $4 \times 6 \mathrm{~cm}$ fluid collection in the left upper quadrant consistent with a pseudocyst and a prominent pancreas suggesting prob- 

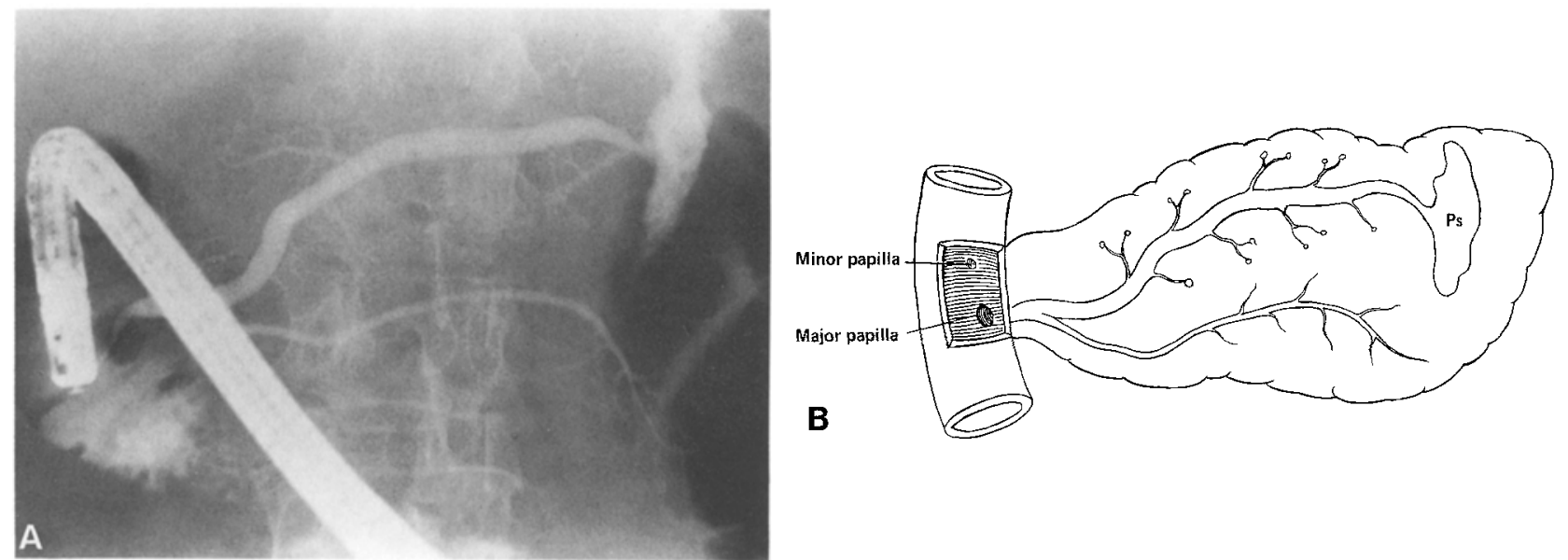

Fig. 1. A An ERCP study shows complete duplication of ductal system extending through the entire length of the pancreas. Both ductal systems were filled simultaneously by 1 injection through the major papilla. The upper ductal system shows dilatation of the main duct, ectasia of the side branches, and a dissecting pseudocyst in the tail region. The lower ductal system is entirely normal. B Schematic drawing of A. Ps, pseudocyst.
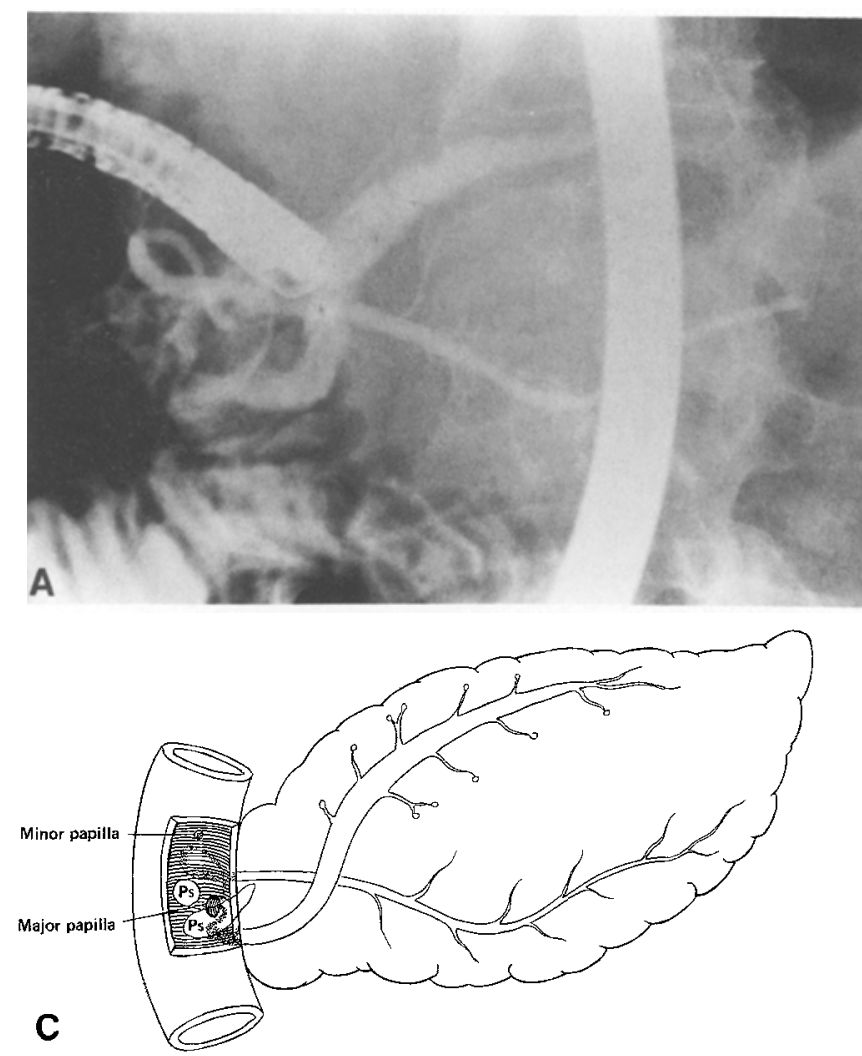

able pancreatitis. A CT scan did not add any other information. Endoscopic retrograde cholangiopancreatography (ERCP) revealed complete duplication of the main pancreatic ductal system. One ductal system showed changes of chronic pancreatitis and a dissecting pseudocyst in the tail region. The second ductal system was entirely normal (Fig. 1). A rudimentary minor papilla was identified but attempts to cannulate it were unsuccessful. The patient was treated conservatively. The pancreatic fistula with pseudocyst in the area of surgical resection gradual-

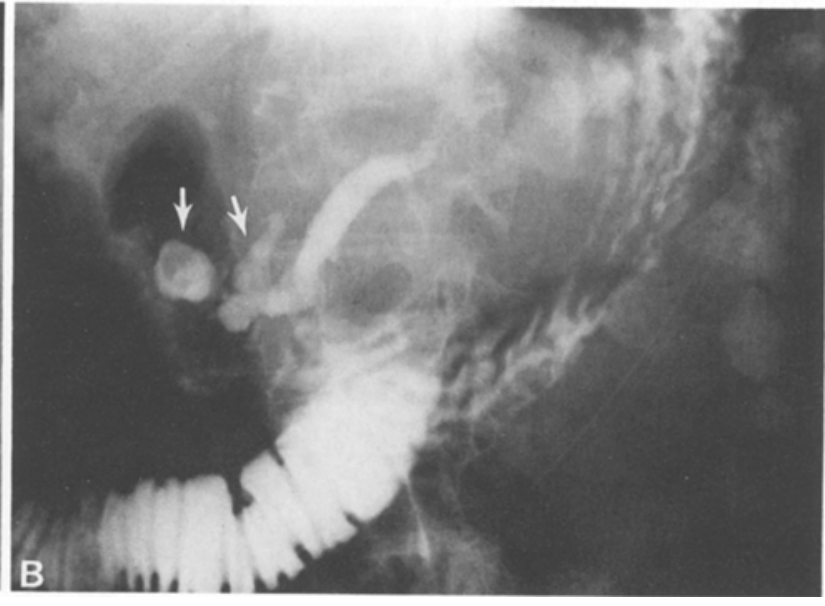

Fig. 2. A An ERCP study shows complete duplication of the ductal system. Both ducts were filled simultaneously by 1 injection through the major papilla. The upper ductal system shows changes of chronic pancreatitis. The lower ductal system is entirely normal. B A film taken 10 minutes later shows significant retained contrast in dilated upper duct. Two small pseudocysts (arrows) in the region of the head are clearly seen. The lower ductal system has completely drained the contrast. C Composite schematic drawing of A and B. Ps, pseudocyst.

ly resolved over 6 weeks. A 1-year follow-up showed no flare of pancreatitis.

\section{Case 2}

A 58-year-old man complained of recurrent abdominal pain and vomiting of $6-9$ months duration. A sonogram revealed a $2 \times 3 \mathrm{~cm}$ pseudocyst in the head of the pancreas. An ERCP 
examination showed complete duplication of the main pancreatic ductal system. One system showed advanced changes of chronic pancreatitis and pseudocysts in the head region. The second ductal system was entirely normal (Fig. 2). Attempts to cannulate the rudimentary minor papilla were unsuccessful. The biliary ductal system was normal. This patient was treated with endoscopic sphincterotomy. At 1 year follow-up there was inprovement in the abdominal pain.

\section{Discussion}

These 2 cases show complete duplication of the main pancreatic duct (duct of Wirsung). This anomaly of the pancreatic ductal system is most unusual and should not be confused with pancreas divisum. The presence of rudimentary minor papilla confirms the presence of original dorsal pancreas that might have undergone regression. Since both ducts communicated at the major papilla and were opacified simultaneously with contrast during injection, we believe that these are ducts of Wirsung. This anomaly can be classified as "duplicated or duplex ventral pancreas." The explanation for this unusual pancreatic ductal anomaly can be hypothesized to be as follows: The 2 buds of the ventral pancreatic anlage persisted and developed into 2 complete ductal systems, both draining the entire adult pancreas via the major papilla. One bud of the ventral pancreatic anlage differentiated and developed into 2 complete ductal systems both draining at the ampulla of Vater via a common channel.

A review of the literature regarding pancreatic ductal variations and ventral pancreas has not revealed any reference to the anomaly of the ventral pancreas as noted in our 2 cases [1-10]. A bifid pancreatic tail presenting as a submucosal gastric mass has been reported previously [11].

The cases of symptomatic pancreas divisum that may simulate our 2 cases clinically and at ERCP findings typically occur in young women with no significant history of alcohol ingestion. In these cases of pancreas divisum the changes of pancreatitis are confined to the dorsal pancreas and can be demonstrated only by cannulation of the minor papilla. The small ventral pancreas is usually normal [12-16].

The incidence of duplex or duplication of the ventral pancreas is not known. Obviously, it must be an extremely rare anomaly. The 2 patients reported here sought medical advice due to symptoms of chronic pancreatitis. Why only 1 ductal system was diseased in both cases is also intriguing and leads us to speculate that this ductal anomaly may predispose to pancreatitis. It seems logical to look for congenital pancreatic ductal anomalies in all patients with chronic recurrent pancreatitis. The widespread use of ERCP may uncover more cases of this unusual pancreatic ductal anomaly.

\section{References}

1. Freeny PC, Lawson TL: Embryology of the pancreatic and biliary duct. In Freeny PC, Lawson TL (eds): Radiology of the Pancreas. New York: Springer-Verlag, 1982, pp 98144

2. Stewart ET, Gennen JE: Development and important relationships of the normal pancreatic and biliary systems. In Stewart ET, Vennes JM, Gennen JE (eds): Atlas of Endoscopic Retrograde Cholangiopancreatography. St. Louis: C.V. Mosby, 1977, pp 33-42

3. Kizu M: Normal endoscopic cholangiopancreatogram. In Takemoto T, Kasugai T (eds): Endoscopic Retrograde Cholangiopancreatography. Tokyo: Igaku-Shoin, 1979, pp 141158

4. Odger PNB: Some observations on the development of the ventral pancreas. $J$ Anat $65: 1-7,1930$

5. Simkins S: Variations in the pancreatic ducts and the minor duodenal papilla. Am J Med Sci 182:626-639, 1931

6. Baldwin WM: The pancreatic ducts in man together with a study of the microscopic structure of the minor duodenal papilla. Anat Rec 5:197-228, 1911

7. Berman LG, Prior JT, Abramow SM, Zeigler DD: A study of the pancreatic ductal system in man by the use of vinyl acetate casts of postmortem preparations, Surg Gynecol $\mathrm{Ob}$ stet 110:391-403, 1960

8. Dawson W, Langman J: An anatomical-radiological study of the pancreatic duct pattern in man. Anat Rec 139:59-68, 1961

9. Kleitsch WP: Anatomy of the pancreas, a study with special reference to the duct system. Arch Surg 71:795-803, 1961

10. Belber JP, Bill K: Fusion anomalies of the pancreatic ductal system, differentiation from pathologic states. Radiology 123:637-642, 1977

11. Kikuchi K, Nomiyami T, Miwa T, Harasawa S, Miwa T: Bifid tail of the pancreas: a case presenting as a gastric submucosal tumor. Am J Gastroenterol 78:23-27, 1983

12. Mitchel CJ, Lintott DJ, Ruddell WSJ, Losowsky MS, Axon ART: Clinical relevance of an unfused pancreatic duct system. Gut 20:1066-1071, 1979

13. Cotton PB: Congenital anomaly of pancreas divisum as cause of obstructive pain and pancreatitis. Gut 21 : 105-114, 1983

14. Gregg JA, Monaco AP, McDermott WV: Pancreas divisum: results of surgical intervention. Am $J$ Surg $145: 488-492,1983$

15. Warshaw AL, Richter JM, Shapiro RH: The cause and treatment of pancreatitis associated with pancreas divisum. Ann Surg 198:443-452, 1983

16. Thompson MH, Williamson RC, Salmon PR: The clinical relevance of isolated ventral pancreas. $B r \quad J \quad S u r g$ 68:101-104, 1981

Received: December 18, 1985; accepted: February 1, 1986 